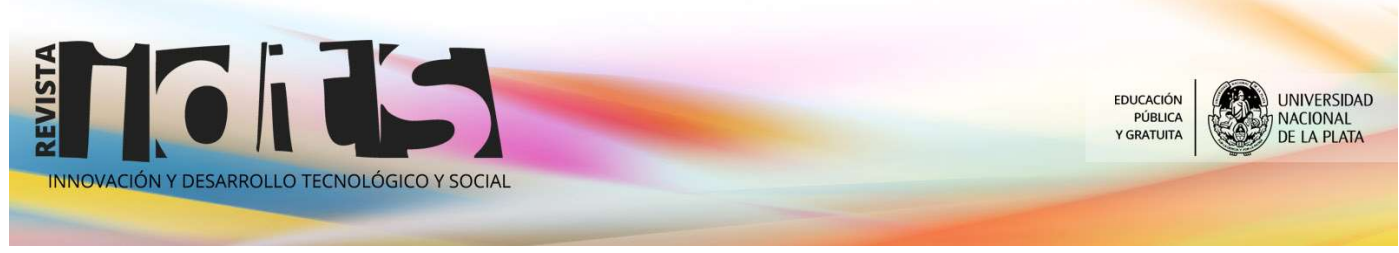

Innovación y Desarrollo Tecnológico y Social (2019) 2: 16-27

\title{
Diseño Gandhiano para el desarrollo de impresoras Braille.
} El aporte desde el Diseño Industrial

\author{
Del Giorgio Solfa, Federico ${ }^{1,2,3}$; Amendolaggine, Guido ${ }^{1,2}$; Tenorio, Florencia ${ }^{1}$; Marozzi, Sofia ${ }^{1}$ \\ ${ }^{1}$ LIDDI, Facultad de Bellas Artes, Universidad Nacional de La Plata. \\ ${ }^{2}$ Comisión de Investigaciones Cientificas de la Provincia de Buenos Aires \\ ${ }^{3}$ delgiorgio@fba.unlp.edu.ar
}

Resumen Se aborda transdisciplinariamente el estudio, diseño y desarrollo de una impresora braille digital de bajo costo. El desafio principal, se centró en reducir significativamente el alto costo de este tipo de impresoras y consecuentemente los servicios de impresión, que sumado a la baja comercialización en el país, dificulta aún más el acceso de estas herramientas esenciales a gran parte de los sectores bajos y medios de la estructura social argentina.

Un grupo interdisciplinario conformado por docentes, graduados y estudiantes de las carreras de diseño industrial e informática de la Universidad Nacional de La Plata, desarrolló el diseño y prototipo de una impresora braille según los principios de la Ingeniería Gandhiana, partiendo de la reutilización de impresoras de chorro de tinta. El proyecto consiste en el diseño, desarrollo y generación de una impresora Braille de bajo costo, de producción local, que prioriza la utilización de componentes de fabricación nacional para su ensamblado.

Para lograr esto se diseñó y fabricó un sistema electrónico y electromecánico para adaptar cualquier impresora Inkjet a los objetivos del proyecto, posibilitando el reciclado de impresoras en desuso. Es decir, se plantea resolver a través de la utilización de impresoras de texto, aplicando conocimientos técnicos de informática y diseño industrial, para adaptar los componentes tecnológicos a la impresión en léxico braille. También se desarrolló, por un lado un software que controle la impresora y permita su uso de manera sencilla y accesible. Por otro lado, se adaptó el hardware y los componentes externos con tecnología de impresión 3D y termoformado, produciendo una carcasa que contiene a la totalidad de la impresora.

Por último, se realizó una sistematización del proceso de ensamblado del prototipo de impresora, posibilitando replicar la misma. Utilizando metodologías del Diseño Gandhiano, se obtuvo un prototipo y dos propuestas alternativas de carcasas. El objetivo principal, que guió a este trabajo, fue que las personas no videntes puedan acceder a impresoras braille de bajo costo y asi promover un impacto favorable en el desarrollo cultural de sus vidas cotidianas.

Palabras claves: diseño Gandhiano; desarrollo de producto ; impresora Braille ; diseño industrial ; reutilización

Recibido 6/7/2019 Aceptado 12/11/2019

DOI: $10.24215 / 26838559 \mathrm{e} 007$

\section{Gandhian design for the development of Braille printers. The contribution of Industrial Design}

\begin{abstract}
The study, design and development of a low-cost digital braille printer is boarded with a transdisciplinary approach. The main challenge was focused on reducing significantly the high cost of this type of printers and their printing services. This context is aggravated with the low commercialization of
\end{abstract}




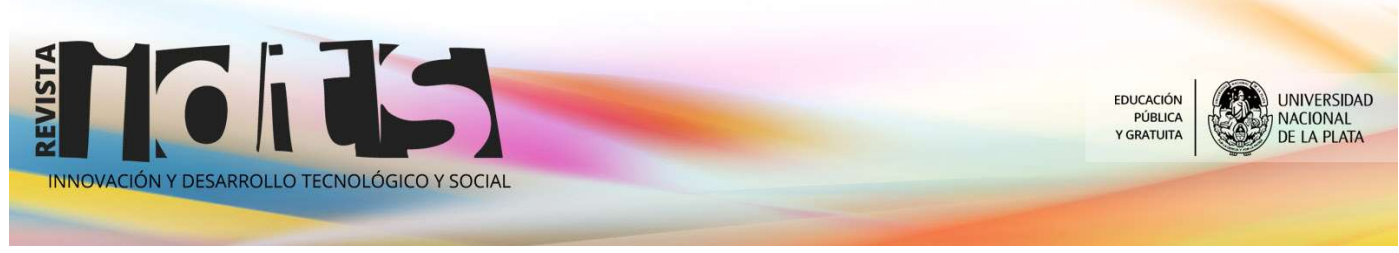

Innovación y Desarrollo Tecnológico y Social (2019) 2: 16-27

these products in the country, a factor that makes the access of these tools -that are essential to much of the low and middle sectors of the Argentine social structure- even more difficult.

An interdisciplinary group of teachers, graduates and students of the careers of industrial design and informatics degree from the National University of La Plata, developed the design and prototype of a braille printer. The work was carried out according to the principles of Gandhian Engineering, based on the reuse of ink-jet printers. The project consists of the design, development and generation of a low-cost Braille printer, produced locally, which prioritizes the use of nationally manufactured components for assembly.

To achieve this, an electronic and electromechanical system was designed and manufactured to adapt any Inkjet printer to the objectives of the project, enabling the recycling of obsolete printers. This means, it is proposed to resolve through the use of text printers, applying technical knowledge of computer science and industrial design, to adapt the technological components to the Braille lexicon printer.

They also developed, on the one hand, software that controls the printer and allows its use in a simple and accessible way. On the other hand, the hardware and external components were adapted with $3 D$ printing technology and thermoforming, producing a housing that contains the entire printer.

Finally, a systematization of the assembly process of the printer prototype was carried out, making it possible to replicate it. Using methodologies of Gandhian Design, a prototype and two alternative proposals of carcasses were obtained. The main objective, which guided this work, was that blind people can access low-cost braille printers promoting by this a favorable impact on the cultural development of their daily lives.

Keywords: Gandhian design ; product development ; braille printer ; industrial design ; reuse

\section{Novedad u originalidad local en el conocimiento:}

El proyecto fue articulado entre las Facultades de Bellas Artes y de Informática de la Universidad Nacional de La Plata (UNLP) ${ }^{1}$, la Escuela de Educación Especial para niños no videntes y la Biblioteca Braille y Parlante.

Este desarrollo de impresora braille de bajo costo, utilizó el concepto de ingeniería gandhiana, que posee una visión íntegramente colaborativa para el desarrollo y producción de objetos a gran escala, de bajo costo y socialmente accesibles (Prahalad y Mashelkar, 2010a y 2010b; Amendolaggine y Del Giorgio Solfa, 2016).

El diseño industrial resolvió la integralidad del producto y la interfaz física; y la Informática, utilizó Arduino ${ }^{2}$ para la programación. Se adaptaron las piezas y se proyectó el prototipo como un kit de ensamblaje a bajo costo (Prahalad y Mashelkar, 2010b).

\footnotetext{
${ }^{1}$ Equipo de Trabajo - Proyectos de Tecnologías para la Inclusión Social (PTIS)

Director Técnico del Proyecto: Federico Del Giorgio Solfa

Graduados: Guido Amendolaggine, Pablo Barbato, Mariel Leguizamon, Miguel Luengo, Fernando Nehele, Antonella Tange, Florencia Tenorio y Adán Mauri Úngaro.

Alumnos: Delfina Aramburu, Gonzalo Armisén, Sofía Belabarba, Leonardo Boschero, Francisco Javier Calzadilla, Joaquín Cavo, Nicolás Epíscopo, Damián Estela, Carolina Galeazzi, Sofía Lara Marozzi, Pablo Miliarchuk, Agustín Parmisano, Ignacio Pórfido, Ángel Ramírez, Camila Ronconi, Estefanía Salomón, Mariana Sirello, Agustina Torá y Pilar Vivas.

${ }^{2}$ Arduino es una organización de código y hardware abiertos, que se presenta como un proyecto de la comunidad internacional, que diseña y fabrica placas para desarrollo de hardware que permiten construir dispositivos digitales e interactivos, para detectar y controlar objetos del mundo real.
} 


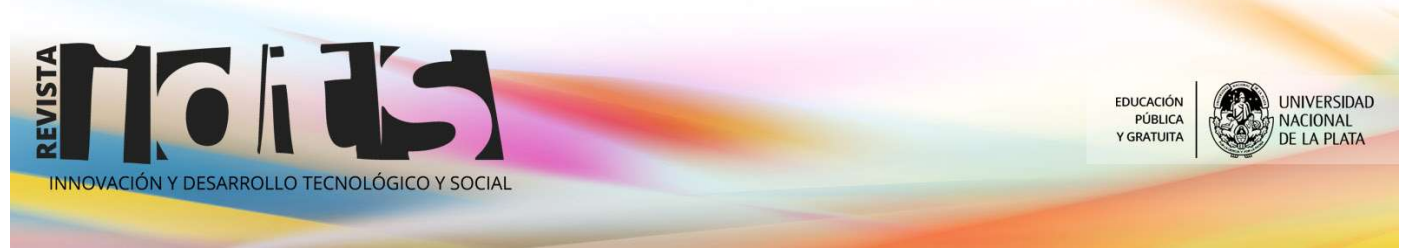

Innovación y Desarrollo Tecnológico y Social (2019) 2: 16-27

\section{Grado de relevancia:}

El proyecto, apuntó a que personas no videntes de sectores medios y bajos (Sémbler, 2006) puedan acceder a impresoras braille de bajo costo y desarrollarse y convivir dignamente en sociedad (Prahalad y Mashelkar, 2010b; Ortiz Ortiz y Matey García, 2011; Amendolaggine et al., 2016).

El principal objetivo fue ampliar el universo de posibilidades de quienes poseen algún tipo de discapacidad visual, permitiéndoles obtener, a partir de una impresora hogareña de chorro de tinta, una impresora braille eficiente y ergonómica, que garantice la seguridad del usuario a un costo considerablemente menor al resto de las que se encuentran en el mercado (Bononi et al., 2015; Esparza-Maldonado, 2018).

El objetivo tecnológico del proyecto, fue diseñar una impresora braille de bajo costo y producción nacional, para ser utilizada de manera pública, gratuita, accesible y pueda ser fácilmente replicable (Prahalad y Mashelkar, 2010b).

Entre los objetivos, se estableció:

- Obtener una impresora braille, reutilizando componentes de impresoras masivas.

- Generar independencia y soberanía tecnológica para su diseño y desarrollo.

- Desarrollar un software que controle la impresora y permita su uso de manera sencilla y accesible.

- Poner la impresora a disposición de las instituciones de la sociedad civil y de usuarios particulares, para su uso de manera accesible, pública y gratuita.

- Promover la inclusión social y la igualdad a sujetos con disminución visual, que no tienen acceso a la impresión braille.

- Vincular la ciencia y tecnología con la comunidad, a través del pensamiento y acción inclusiva, aplicada a resolver problemáticas sociales.

\section{Grado de pertinencia:}

Estudios realizados en Argentina, muestran que: "el 7,1\% del total de discapacitados visuales son ciegos" (García et al., 2014: p.4); además, existen prevalencias de ceguera de 1,1\% en áreas urbanas (Nano et al., 2006) y 1,8\% en áreas urbanas y rurales, en mayores de 50 años (Barrenechea et al., 2013).

Las impresoras braille que pueden adquirirse en el mercado argentino, son de origen importado. Sus precios son significativamente elevados, haciendo imposible el acceso por parte de personas no videntes, cuyas familias poseen ingresos medios y bajos.

Según cifras de la Organización Mundial de la Salud, en el año 2012 había en el mundo aproximadamente 285 millones de personas con discapacidad visual, de las cuales 39 millones eran ciegas y 246 millones presentaban baja visión. Aproximadamente, un $90 \%$ de las personas con discapacidad visual se concentra en los países en desarrollo. Por tanto, haciendo un cálculo aproximado, podemos decir que el número de potenciales lectores de braille rondaría los 50 millones de personas. (ONCE, 2015, p. 28). 


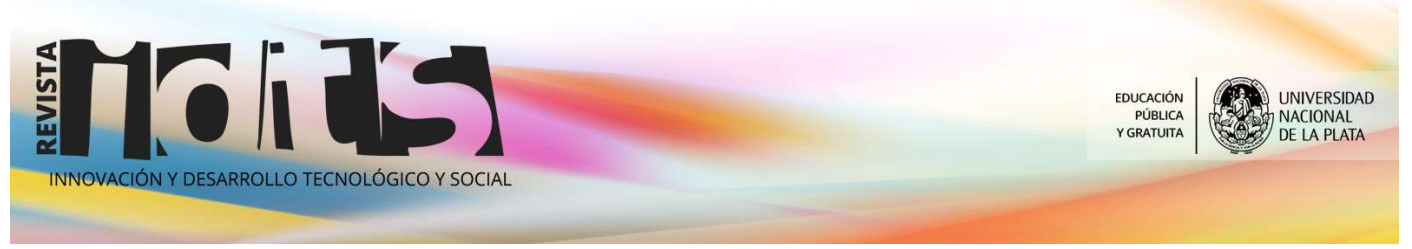

Innovación y Desarrollo Tecnológico y Social (2019) 2: 16-27

Son muchas las circunstancias en las que los ciegos no disponen de textos en braille, situándolos en desventaja, respecto al resto de la población. No tener acceso a la información escrita, provoca dependencia de terceras personas para resolver situaciones cotidianas: alimentación, informes médicos, rutas turísticas, etcétera (ONCE, 2015).

La solución que el proyecto asumió dar, si bien es una respuesta acotada para una zona del Partido de La Plata, la misma se diseña con la idea de poder replicarse en otros barrios y localidades.

\section{Grado de demanda:}

La impresora beneficiaría a aproximadamente cincuenta personas no videntes de la ciudad de La Plata, que se constituyen en potenciales usuarios, pudiendo ser ampliada mediante vínculos con instituciones para ciegos.

Con la puesta en marcha de la Edición 2014 del Hackatón (maratón de desarrollo colaborativo), en el día internacional de las personas con discapacidad, la UNLP, a través de la Secretaría de Relaciones Institucionales, la Comisión Universitaria sobre discapacidad (CUD) y el Centro Superior para el Procesamiento de la Información (CeSPI), se organizaron las Jornadas: "Accesibilidad y Universidad. Ideas para la Acción".

En ese marco, se presentó en el Rectorado de la UNLP, una muestra con los proyectos y acciones que, las Facultades y dependencias, llevaban a cabo sobre temas de discapacidad y accesibilidad (Facultad de Informática, 2017).

Las presentación de la impresora de código Braille desarrollada por un grupo de estudiantes de la Facultad de Informática, fue muy bien recibida por el público, impactando la noticia en diversos medios locales y nacionales (Radio Universidad, 2014; Télam, 2014; Página/12, 2014; TVP, 2015).

Con el acompañamiento de los medios, el contacto con las organizaciones de la sociedad civil resultó más fluido y a partir de la convocatoria 2015, a presentar propuestas para los Proyectos de Tecnologías para la Inclusión Social (PTIS) del Ministerio de Ciencia, Tecnología e Innovación Productiva de la Nación, se formalizó una participación conjunta con la Asociación Civil América Grande, quien fue la entidad solicitante y administradora de los fondos otorgados con la acreditación del proyecto (TVP, 2015).

\section{Desarrollo del producto}

En el marco de las veinticuatro horas de Accesibilidad, para diseñar y desarrollar aplicaciones (Hackatón edición 2014), que organizó la Dirección de Innovación Tecnológica de la UNLP junto a la Facultad de Informática, surgió la idea inicial y el desarrollo del primer plan de trabajo (Facultad de Informática, 2017).

Así nació el primer prototipo de impresora en sistema braille, pero funcionaba de manera lenta. Sus componentes eran muy precarios, la electromecánica y electrónica estaban desprotegidas, no poseía un software que permitiera el control por parte de invidentes y la seguridad en general era inadecuada. 


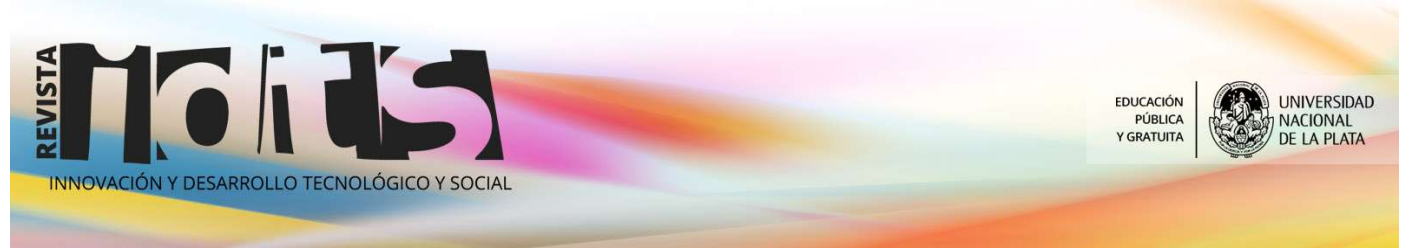

Innovación y Desarrollo Tecnológico y Social (2019) 2: 16-27

Es por eso que surge la necesidad de seguir profundizando el desarrollo de la misma, mejorar su calidad y sistematizar el proceso, para que la permita replicar la experiencia y multiplicar los resultados.

El proyecto integral de diseño y desarrollo de la impresora de código braille de bajo costo (concepto de diseño gandhiano), se llevó a cabo por un grupo de docentes, graduados y estudiantes de Diseño Industrial, con asiento en el Laboratorio de Investigación y Desarrollo Tecnológico del Diseño Industrial (LIDDI) y en el marco de un acuerdo cooperativo entre las Facultades de Bellas Artes e Informática de la UNLP (Prahalad y Mashelkar, 2010a y 2010b; Amendolaggine y Del Giorgio Solfa, 2016; Amendolaggine et al., 2016; Departamento de Diseño Industrial, 2016).

En una primera reunión, se realizaron los primeros acuerdos, en cuanto a días y horario de trabajo, división de tareas, entre otras cuestiones. También se realizaron reuniones de planificación y seguimiento, donde los distintos miembros del equipo pusieron en común los avances realizados y se planificaron ajustes en el plan de trabajo original.

Para comprender el proceso de diseño que se llevó a cabo, nos basaremos en el modelo de doble diamante, desarrollado por el Design Council en 2005, el cual divide la secuencia en cuatro etapas fundamentales: 1. Descubrir, 2. Definir, 3. Desarrollar, y 4. Entregar. Las dos primeras corresponden a la interpretación y definición de la problemática con la que se está trabajando. Las siguientes dos, corresponden al desarrollo de soluciones y sus respectivas pruebas, hasta llegar a la solución final y su puesta en funcionamiento (Design Council, 2007).

\section{Fase 1: Descubrir}

Esta es la fase inicial y más importante del proceso, ya que trata de conocer y comprender en profundidad la problemática a abordar. Se caracteriza por ser una etapa divergente, en la cual se debe acudir a diferentes fuentes para recolectar la mayor cantidad de información posible, que se convertirán en los cimientos del nuevo desarrollo.

En este proyecto, fue una etapa de investigación y caracterización de los usuarios con este tipo de discapacidad. Se acopió información relacionada y se efectuó un relevamiento de los productos existentes en el mercado. La investigación sobre la problemática, alcanzó los contextos: nacional, regional e internacional.

Las primeras conclusiones sobre lo investigado y relevado, permitió plantear un plan de trabajo acorde a la idea rectora y a los requerimientos funcionales que debía alcanzar el producto.

En este caso, se llevaron a cabo tres líneas de investigación en paralelo. Por un lado, el trabajo de campo, visitando entidades públicas para personas no videntes, como escuelas especiales y bibliotecas braille, con el objetivo de comprender realidad y contexto de estas personas.

Por el otro, se realizó una revisión de literaturas específicas, relacionadas con la ergonomía, legislaciones vigentes, tecnologías aplicables, casos de estudio, entre otras. También, se llevó a cabo un relevamiento de mercado, para reconocer los productos disponibles, sus costos y alcances funcionales.

Finalmente, se seleccionó una impresora comercial de chorro de tinta y se aplicó ingeniería inversa, para relevar la totalidad de partes, entender su lógica de funcionamiento y analizar que componentes podrían utilizarse o ser adaptados para la nueva impresora braille. 


\section{Fase 2: Definir}

Esta segunda fase, se caracteriza por un minucioso análisis cualitativo de la información resultante de la etapa anterior, que permite definir con precisión el problema y sus implicancias, tales como: requerimientos, requisitos, limitaciones, normativa legal relacionada y otras. El objetivo es definir el brief, que va a acompañar y dar forma al proyecto.

La Facultad de Informática, en el marco del "Hackatón Tecnológico por la Discapacidad"3 , había desarrollado un prototipo de impresora braille (Openbraille, 2014). Este primer desarrollo, tuvo una gran difusión en los medios de comunicación y en los ámbitos académicos, generando amplia expectativa en diversos actores tanto estatales como privados y en las instituciones para novidentes, las cuales en su mayoría cuentan con impresoras importadas de alto costo (TVP, 2015).

La aparición de una demanda concreta de instituciones, escuelas y organismos que engloban a esta población, motivó la vinculación entre ambas Facultades. Sobre todo, debido a las características del prototipo existente (el cual se reducía a la programación, circuitos y conexiones a la vista) y lo hacían inviable para la utilización inmediata de los usuarios. Por esta razón, se inició un trabajo conjunto con el Departamento de Diseño Industrial de la UNLP.

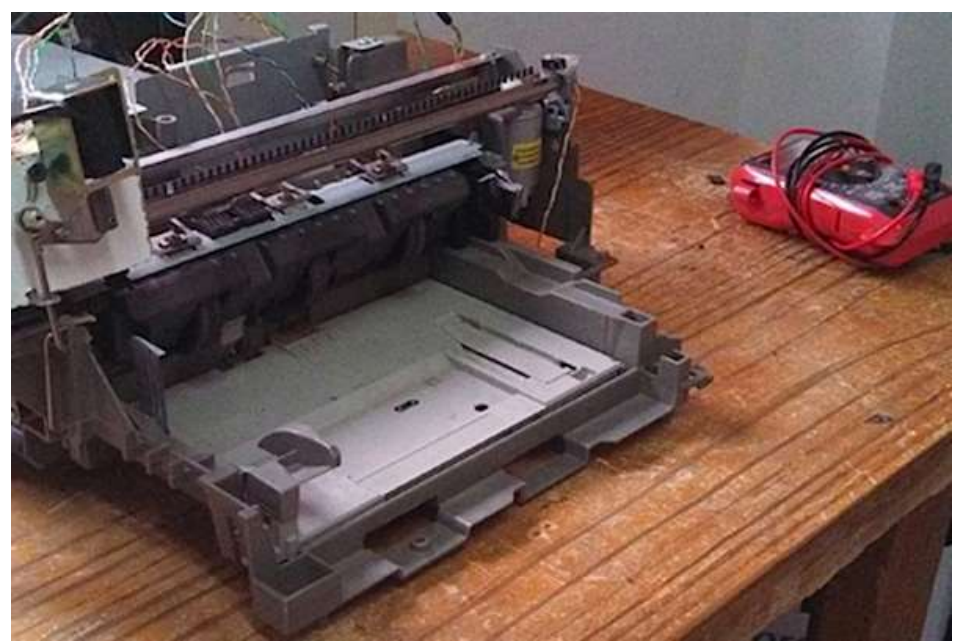

Figura 1. Primer prototipo de impresora braille (Openbraille, 2014)

Figure 1. First braille printer prototype (Openbraille, 2014)

Si bien, el proyecto se desarrolló en el transcurso de dos años, entre 2015 y 2017, existió una etapa previa a la financiación del Ministerio de Ciencia, Tecnología e Innovación productiva de la Nación (ahora Secretaria), a donde se definió el anteproyecto, a partir de varios encuentros entre estudiantes y graduados de ambas Facultades.

\footnotetext{
${ }^{3}$ Véase: https://unlp.edu.ar/comisiondiscapacidad/el-cespi-coorganiza-el-hackaton-tecnologico-por-la-discapacidad$\underline{9167}$
} 


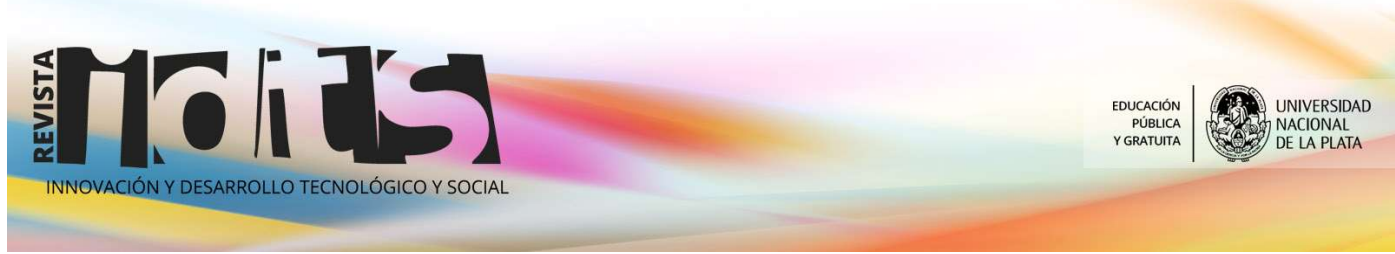

Innovación y Desarrollo Tecnológico y Social (2019) 2: 16-27

\section{Fase 3: Desarrollar}

La tercera fase tiene que ver con el diseño en sí mismo, es donde se desarrolla el producto y sus posibles alternativas, para alcanzar un producto que mejor satisfaga las necesidades predefinidas, en este caso las de las personas no videntes.

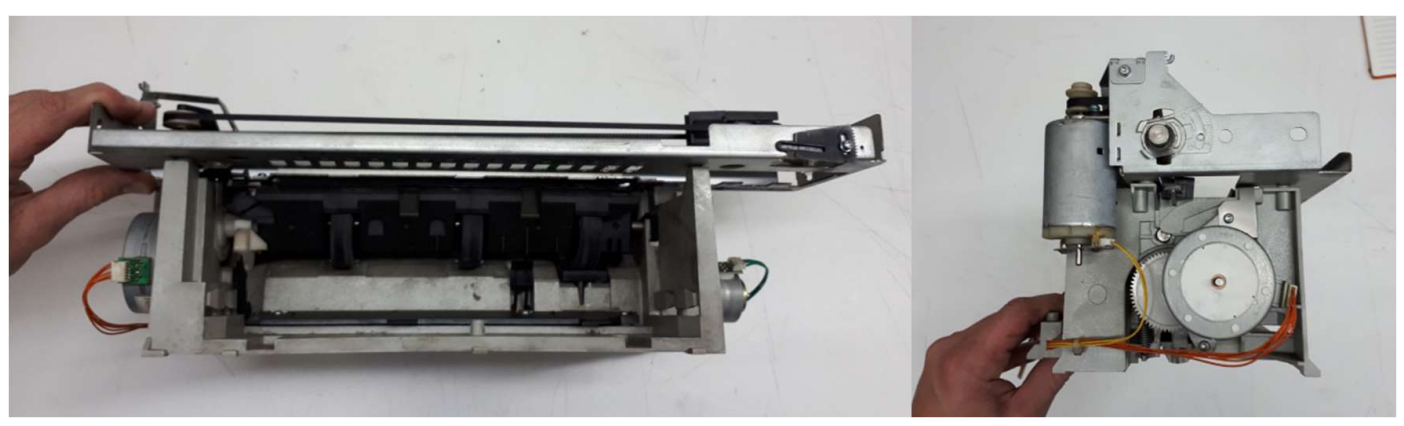

Figura 2. Estructura y paquete electromecánico reciclado de impresora inkjet Figure 2. Structure and recycled electromechanical package of inkjet printer

Seguidamente, se enumeran los principales grupos de actividades llevados a cabo.

1. Diseño e implementación del circuito electrónico. Diseño e implementación del hardware de la impresora. Consiste en la adaptación de la impresora Inkjet y el ensamble con los componentes internos específicos que requiere. Hardware que perfore hojas en sistema braille.

2. Diseño de carcasas. Desarrollo de alternativas morfológicas, tecnológicas y modo de uso, con modos de representación 2D y 3D (fig. 3). Selección y definición de modos de producción, vínculos, oscilaciones morfológicas, relieves y textura.

3. Generación de alternativas en el modo de uso de la impresora. Ajuste de detalles, modelo del producto en tres dimensiones para el desarrollo de las matrices para su fabricación.

4. Pruebas con materiales y procesos alternativos. Utilización de materiales ecológicos.

5. Mejora de las características del producto. Reducción de mecanizados y acabados superficiales, reduciendo costos de fabricación.

6. Fabricación de matriz. Verificación de factibilidad de la carcasa con el proceso de termoconformado (Vacuum Forming), y procesos alternativos que deriven de la investigación sobre los antecedentes del producto. Como también la impresión de las piezas necesarias en tecnología de impresión 3D. Aquí es fundamental la experimentación y el prototipado constante, ya que a través de un proceso de prueba y error consciente, se van perfeccionando las ideas hasta llegar a la mejor solución posible. Es por eso que se decide producir las piezas con impresión 3D, bajo la misma lógica RepRap, es decir código abierto, descarga e impresión libre de las piezas.

7. Proceso productivo y ensamble de la carcasa. Termoformado de las carcasas, control, análisis y corrección de la matriz para el ajuste y adecuado encastre de las carcasas.

8. Ajuste general del producto. Perfeccionamiento de los componentes finales. Realización de las primeras experiencias de uso con usuarios videntes y no videntes.

9. Diseño de software. Diseño e implementación del software de control de la impresora.

9.1. Software de control de la impresora. Interfaz dual de fácil acceso y sencilla utilización, tanto para personas no videntes como videntes. 


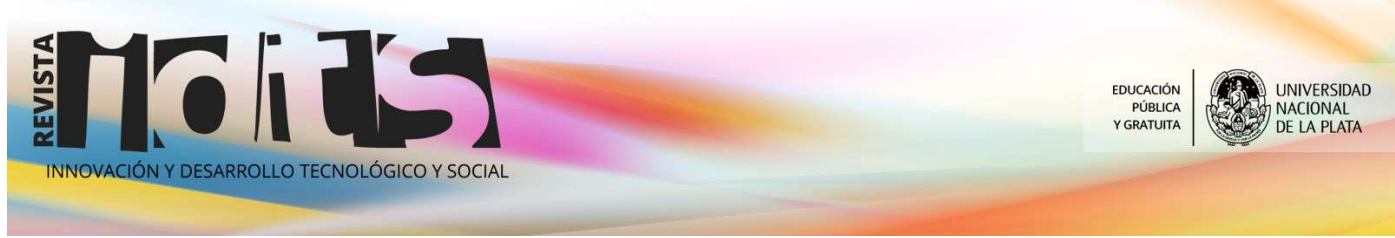

Innovación y Desarrollo Tecnológico y Social (2019) 2: 16-27

9.2. Diseño de un sistema voz. Diseño, desarrollo e implementación de un sistema de voz que guíe a los usuarios con problemas visuales en la configuración de la voz sistema y el uso. Sistema voz complementario al software de control que guíe a la impresora.

10. Ensamble de componentes internos $y$ carcasa. Ensamblado del hardware (componentes internos), carcasa y conexión del software. Obtención de la impresora ensamblada y lista para su utilización.

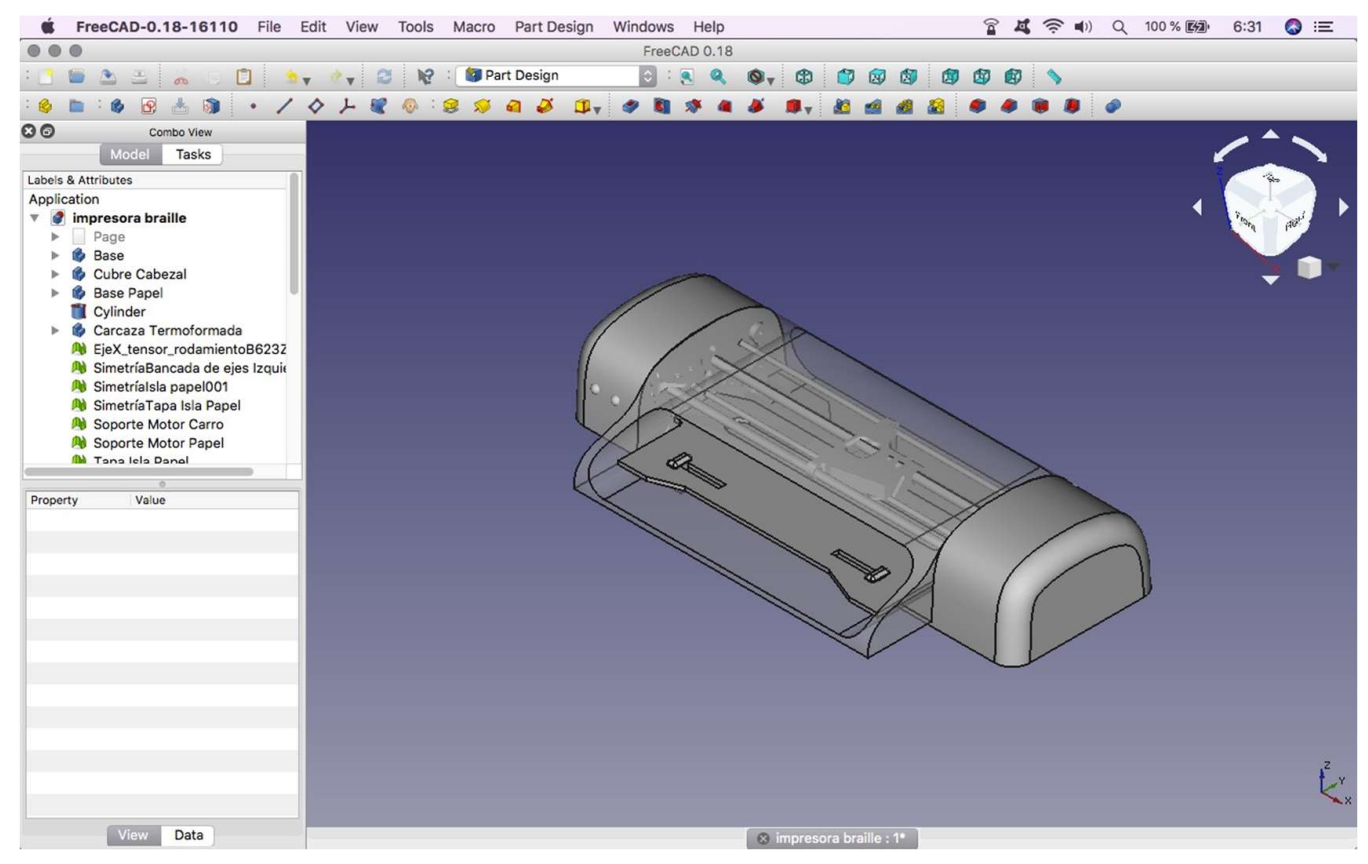

Figura 3. Modelización del diseño de los componentes con FreeCAD

Figure 3. Modeling the design of components with FreeCAD

\section{Fase 4: Entregar}

Esta es la fase final del proceso, en la cual se arriba al diseño definitivo y su prototipo, sobre el cual se realizan las pruebas finales. Si resulta exitoso, el último paso es poner en producción el diseño, introducir el producto en el mercado, liberando y difundiendo las características técnicas y modos de producción. 
Innovación y Desarrollo Tecnológico y Social (2019) 2: 16-27

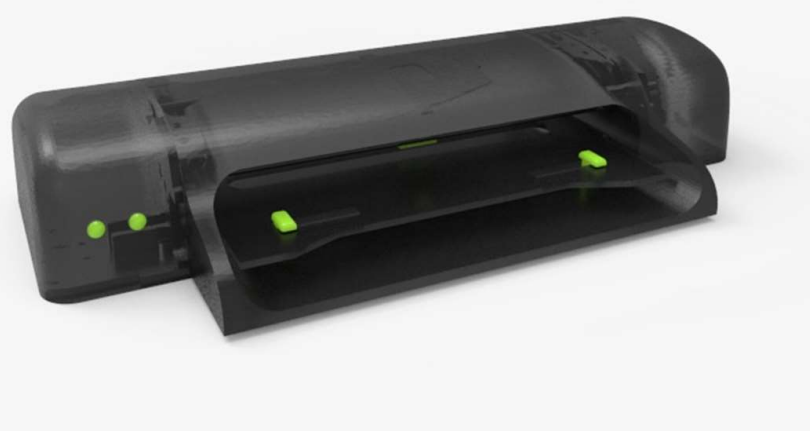

Figura 4. Impresora braille de bajo costo con componentes reciclados

Figure 4. Low cost braille printer with recycled components
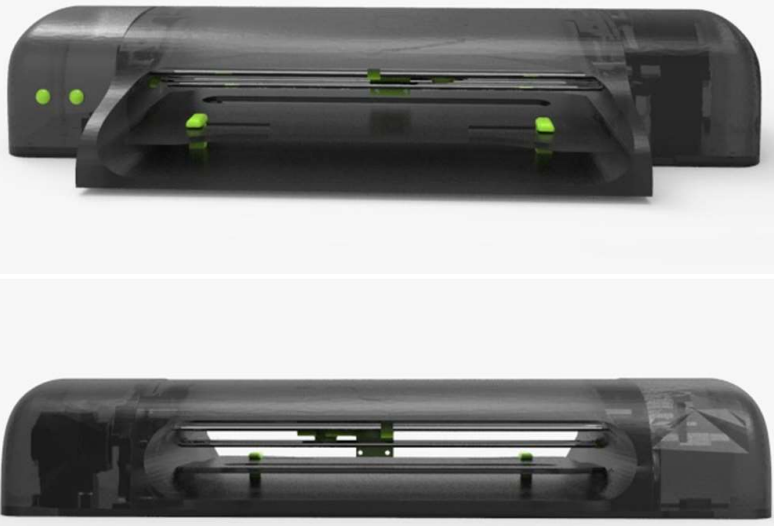

Figura 5. Vistas frontal y trasera de la Impresora braille de bajo costo Figure 5. Front and back views of the low cost braille printer
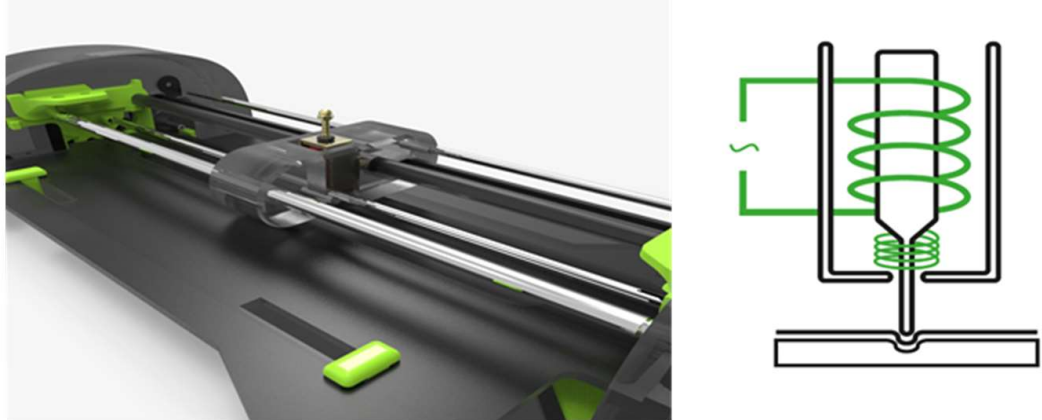

Figura 6. Cabezal electropunzón y detalle del solenoide Figure 6. Electro-puncture head and solenoid detail 


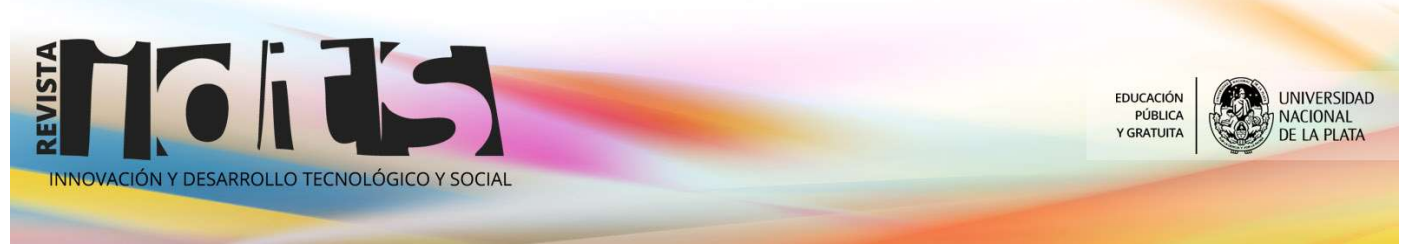

Innovación y Desarrollo Tecnológico y Social (2019) 2: 16-27

Seguidamente, podemos observar un comparativo de los costos de producción entre las dos alternativas diseñadas y las opciones comerciales relevadas de origen sueco $^{4}$.

Tabla 1. Comparativo entre impresoras braille analizadas y diseñadas

Table 1. Comparison between braille printers analyzed and designed

\begin{tabular}{lccccc}
\hline Marca & Modelo & Impresión & Velocidad (A4) & Ruido & Precio (USD) \\
\hline INDEX & Basic-D V5 & Doble & $350 / \mathrm{h}$ & Muy alto & 3.760 \\
INDEX & Everest-D V5 & Doble & $400 / \mathrm{h}$ & Muy alto & 4.788 \\
UNLP & Reciclada & Simple & $60 / \mathrm{h}$ & Medio & 150 \\
UNLP & Nueva & Simple & $60 / \mathrm{h}$ & Medio & 380 \\
\hline
\end{tabular}

Con ello demostramos y concluimos, que es factible el desarrollo de dos tipos de impresoras braille de bajo costo, una basada en la reutilización de impresoras inkjet (inyección de tinta) y otra producida con nuevos componentes.

Como corolario, tenemos la comprobación de que el diseño gandhiano es posible de aplicar y que puede dar una respuesta concreta a la necesidad de impresión braille doméstica a bajo costo.

Finalmente, además afirmamos, que la experiencia recogida a lo largo de este proyecto ha enriquecido a alumnos, graduados y docentes de diseño industrial e informática. Por otro lado, estas disciplinas han logrado un nuevo reconocimiento de su potencial conjunto, por parte de las organizaciones involucradas y de las entidades del Estado que apoyaron esta iniciativa, y de la sociedad en general.

\section{Información sobre el patentamiento/registro de la innovación o desarrollo}

La totalidad del proyecto es de código abierto, tanto partes y carcasas, como software. Se provee el conjunto de planos CAD 3D de cada parte. En cuanto al software que hace funcionar a la máquina, se trata de un programa open source (código abierto). Esto permite a cualquier usuario en cualquier parte del mundo pueda utilizar, cambiar, o mejorar el diseño y desarrollo y redistribuirlo, ya sea en su forma modificada o en su forma original.

\section{Financiamiento}

Este proyecto fue acreditado en el 2015, en la presentación de Proyectos de Tecnologías para la Inclusión Social (PTIS) del Programa Consejo de las Demandas de Actores Sociales (PROCODAS), organizado por la Subsecretaria de Políticas en Ciencia, Tecnología e Innovación Productiva (Disposición SSPCTIP No 25/2015) del Ministerio de Ciencia, Tecnología e

\footnotetext{
${ }^{4}$ https://www.indexbraille.com/
} 


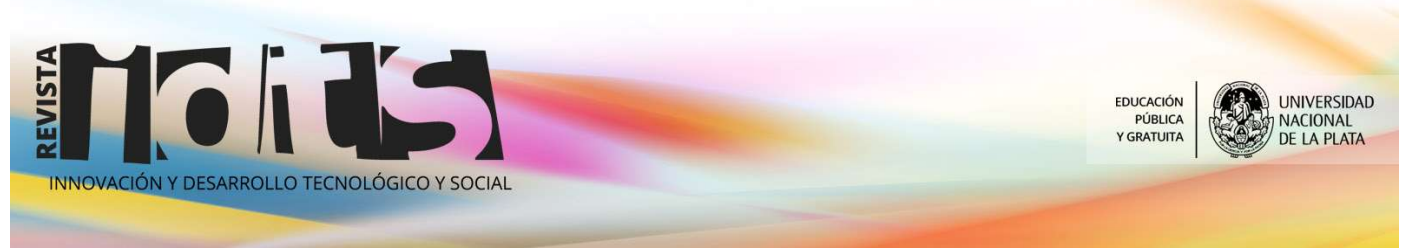

Innovación y Desarrollo Tecnológico y Social (2019) 2: 16-27

Innovación Productiva de la Nación (Resolución MinCTIP N ${ }^{\circ}$ 620/2015) y contó con un financiamiento total de $\$ 69.593,80$ (aprox. USD 7.520).

\section{$\underline{\text { Agradecimientos }}$}

Agradecemos a las siguientes instituciones por confiar la labor al equipo de trabajo:

- Biblioteca Braille y Parlante de la Provincia de Buenos Aires

- Escuela Especial No 515 "Lic. Elina Tejerina de Walsh"

\section{Referencias bibliográficas}

Amendolaggine, G., Nehele, F., Tange, A., y Tenorio, F. (2016). Diseño e Inclusión Social. Desarrollo de una impresora braille de bajo costo. Actas de las VIII Jornadas de Investigación en Disciplinas Artísticas y Proyectuales (JIDAP) de la UNLP, Argentina. Recuperado de http://hdl.handle.net/10915/57063

Amendolaggine, G., y Del Giorgio Solfa, F. (Octubre, 2016). Diseño industrial e ingeniería gandhiana: modelo de diseño y desarrollo local basado en productos masivos de bajo costo. VIII Jornadas de Investigación en Disciplinas Artísticas y Proyectuales. Facultad de Bellas Artes de la Universidad Nacional de La Plata. Recuperado de http://digital.cic.gba.gob.ar/handle/11746/10062

Barrenechea, R., De La Fuente, I., Plaza, R.G., Flores, N., Segovia, L., Villagómez, Z., Camarero, E.E., Zepeda-Romero, L.C., Lansingh, V.C., Limburg, H., y Silva, J. C. (2015). Encuesta nacional de ceguera y deficiencia visual evitable en Argentina, 2013. Recuperado de http://hdl.handle.net/20.500.12104/66398

Bononi, J., Carvalho, J.A., Domiciano, C.L.C., Pinheiro, O.J., Paschoarelli, L.C., y Medola, F.O. (2015). Aspects inclusive fashion design for children with blindness. $15^{\circ}$ ERGODESIGN Congresso Internacional de Ergonomia e Usabilidade de Interfaces Humano-tecnologia I $15^{\circ}$ USIHC - Congresso Internacional de Ergonomia e Usabilidade de Interfaces Humanocomputador, Blucher Design Proceedings, Vol. 2, pp. 954-966. ISSN 2318-6968. Recuperado de www.proceedings.blucher.com.br/article-details/19049

Departamento de Diseño Industrial, FBA-UNLP (2016). Informe institucional 2015-2016, Tableros ( $\left.{ }^{\circ} 7\right)$, pp. 75-84, octubre 2016. ISSN 2525-1589.

Design Council (2007). Eleven lessons: managing design in eleven global brands. A study of the design process. Londres. Recuperado de https://www.designcouncil.org.uk/sites/default/files/asset/document/ElevenLessons_Design_Co uncil\%20(2).pdf

Esparza-Maldonado, A.L., Margain-Fuentes, L.Y., Álvarez-Rodríguez, F. J., y BenítezGuerrero, E. I. (2018). Desarrollo y evaluación de un Sistema Interactivo para personas con discapacidad visual. TecnoLógicas, 21(41), pp. 149-157. Recuperado de http://www.scielo.org.co/pdf/teclo/v21n41/v21n41a10.pdf 


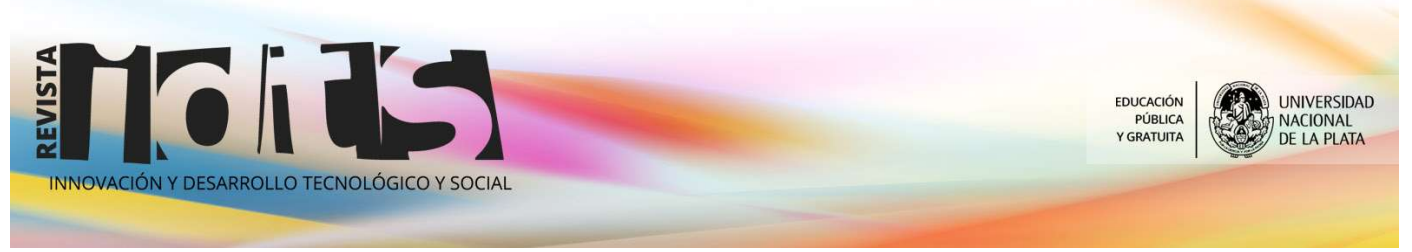

Innovación y Desarrollo Tecnológico y Social (2019) 2: 16-27

Facultad de Informática, UNLP (Secretaría de Extensión e Innovación) (2017). Hackatón accesibilidad o maratón de desarrollo. Recuperado de http://extension.info.unlp.edu.ar/article/printPreview/id/360

García, M.G., Frías, M., Leonhardt, L., Junquera, P. (2014). Calendario Inclusivo Braille y Tinta. Buenos Aires, UADE. Recuperado de https://repositorio.uade.edu. ar/xmlui/handle/123456789/4072

Mashelkar, R.A. (2012). On building an inclusive innovation ecosystem. Conference on Innovation for Inclusive Development.

Nano, M.E., Nano, H.D., Mugica, H.M., Silva, J.C., Montaña, G., y Limburg, H. (2006). Rapid assessment of visual impairment due to cataract and cataract surgical services in urban Argentina. Ophthalmic Epidemiol, 13(3), pp. 191-197.

Openbraille (2014). Adaptación de impresoras de chorro a tinta y matriciales con hardware y software libre para generar Código Braille de muy bajo costo. Recuperado de http://www.openbraille.com.ar/index.php

Organización de las Naciones Unidas (ONU) (2006). Convención sobre los Derechos de las Personas con Discapacidad. Recuperado de https://www.un.org/esa/socdev/enable/documents/tccconvs.pdf

Organización Nacional de Ciegos Españoles (ONCE), Comisión Braille Española. (2015). La Didáctica del Braille más allá del código. Nuevas perspectivas en la alfabetización del alumnado con discapacidad visual. Documento técnico B11, v. 1

Ortiz Ortiz, P., y Matey García, M.A. (Eds.) (2011). Discapacidad visual y autonomía personal. Enfoque práctico de la rehabilitación. Madrid, ONCE. Recuperado de http://sid.usal.es/idocs/F8/FDO26230/discap_visual.pdf

Página/12 (Sección Sociedad). (2014). En Braille y accesible. Recuperado de https://www.pagina12.com.ar/diario/sociedad/3-261228-2014-12-04.html

Prahalad, C.K., y Mashelkar, R.A. (2010a). Erfinderische Inder. Harvard Business Manager, pp. 92-104.

Prahalad, C.K., y Mashelkar, R.A. (2010b). Innovation's holy grail. Harvard Business Review, $88(7 / 8)$, pp. 132-141.

Radio Universidad Nacional de La Plata (2014). Impresora Braille a bajo costo. Recuperado de https://www.radiouniversidad.unlp.edu.ar/impresora-braille-a-bajo-costo/

Sémbler R., C. (2006). Estratificación social y clases sociales. Una revisión analítica de los sectores medios. Serie Políticas Sociales, 125. Santiago de Chile, CEPAL.

Télam (2014). Desarrollaron en la UNLP una impresora braille de bajo costo con componentes reciclados. Recuperado de http://www.telam.com.ar/notas/201412/87318-impresora-braillecomponentes-reciclados-unlp.html

Televisión Pública Argentina (TVP). (Visión 7). (2015). Desarrollo argentino de una impresora braille [Video]. Recuperado de https://youtu.be/X5eAvYVtOvI 